\title{
Hybrid Optimization of a Valveless Diaphragm Micropump Using the Cut-Cell Method
}

\author{
D. Kapsoulis, K. Samouchos, X. Trompoukis and K. Giannakoglou \\ Parallel CFD \& Optimization Unit, School Mech. Eng., National Technical University of Athens, Athens 15780, Greece
}

\begin{abstract}
This paper presents the optimization of 3D valveless diaphragm micropump for medical applications. The pump comprises an inlet and outlet diffuser connected to the main chamber equipped with a periodically moving diaphragm that generates the unsteady flow within the device. The optimization, which is related exclusively to the diaphragm motion, aims at maximizing the net flowrate and minimizing the backflow at the outlet diffuser. All CFD analyses are performed using an in-house cut-cell method, based on the finite volume approach, on a many-processor system. To reduce the optimization turn-around time, two optimization methods, a gradient-free evolutionary algorithm enhanced by surrogate evaluation models and a gradient-based (GB) method are synergistically used. To support the GB optimization, the continuous adjoint method that computes the gradient of the objectives with respect to the design variables has been developed and programmed. Using the hybrid optimization method, the Pareto front of non-dominated solutions, in the two-objective space, is computed. Finally, a couple of optimal solutions selected from the computed Pareto front are re-evaluated by considering uncertainties in the operating conditions; these are quantified using the polynomial chaos expansion method.
\end{abstract}

Key words: Diaphragm micropump, cut-cell method, hybrid optimization, adjoint method, evolutionary algorithm, uncertainty quantification.

\section{Introduction}

Diaphragm micropumps [9] are frequently used as medical analysis devices, in biochemical-processing applications [1] such as chemotherapy or for delivering insulin to patients suffering from diabetes [9]. Recently, they are also used in mass spectrometer systems in lightweight spacecrafts or for lab-on chip cooling [10], etc. At least in medical applications, valved pumps are usually avoided due to the high pressure drop across the valves, their reduced lifetime and the damage they may cause to sensitive fluids. Therefore, herein, a valveless pump, in which valves are replaced by diffusers, is designed. Unfortunately, during the operation of valveless diaphragm pumps, back-flow might instantaneously occur at the outlet, being undesirable in drug injection and several other medical applications. Therefore, a design-optimization

Corresponding author: Konstantinos Samouchos, Mechanical Engineer, PhD Student, research fields: CFD, aerodynamics, turbomachinery, and optimization. of such a micropump should target the maximum, or occasionally the desired, net flowrate per period, by also minimizing the exit back-flow. This is presented in this paper, for a micropump with a chamber having fixed dimensions by exclusively controlling the diaphragm motion.

The optimization repetitively makes use of an in-house, adequately validated CFD code based on the cut-cell method [7]. Starting point is a coarse Cartesian grid entirely enclosing the flow domain. Stationary and moving boundaries intersect the background grid which is refined close to the boundaries, at each time-step, so as to increase the accuracy of the flow simulation. A first reason for having selected the cut-cell method is to overcome the use of costly body-fitted grid deformation tools. Another reason is in view of the scheduled extension of this design tool to valved diaphragm pumps, equipped with butterfly valves that fully suppress backflow.

In such a two-objective optimization, an evolutionary algorithm (EA) primarily seeks the 
Pareto front of optimal solutions. Since a high-fidelity/high-cost unsteady flow solver is used, the optimization must be carried out by running the minimum number of CFD evaluations. To this end, the EA implements surrogate evaluation models or metamodels and other techniques as described below. During the population-based stochastic optimization method, an intercalary GB improvement step is regularly carried out for some of the non-dominated solutions at the end of each generation. Combining both methods gives rise to a hybrid (gradient-free and gradient-based) optimization method, an indispensable part of which is the computation of the objective function gradients by means of the continuous adjoint method. Having computed the Pareto front of optimal solutions, an investigation of the effect uncertainties related to the diaphragm motion due to operating/manufacturing imperfections is carried out. The effect these uncertainties have on (some of) the computed optimal solutions is studied by computing the first two statistical moments of the objective functions, using a non-intrusive polynomial chaos expansion (PCE) [11] technique.

\section{Flow Equations \& Grid Generation}

The Navier-Stokes equations governing the 3D unsteady laminar flow of an incompressible fluid,

$$
\Gamma_{i j}^{-1} \frac{\partial V_{j}}{\partial \tau}+\frac{\partial U_{i}}{\partial t}+\frac{\partial f_{i k}}{\partial x_{k}}=0
$$

(for $i=1,4$; repeated indices imply summation) are numerically solved. Index $k$ refers to the Cartesian directions $\left(x_{1}, x_{2}, x_{3}\right)$ with the corresponding velocities denoted by $\left(u_{1}, u_{2}, u_{3}\right)$. The above equation includes the (real) time $(t)$ derivative of $\vec{U}=\left[\begin{array}{llll}0 & u_{1} & u_{2} & u_{3}\end{array}\right]^{T}$ and the pseudo-time $(\tau)$ derivative of $\vec{V}=\left[\begin{array}{lll}p & u_{1} u_{2} & u_{3}\end{array}\right]^{T}$ (where $p$ is the pressure divided by the constant fluid density) according to the pseudo-compressibility solution method [7]. $\vec{f}_{k}$ is the inviscid and viscous fluxes; the latter depends on the viscous stresses $\tau_{i k}=v\left(\frac{\partial u_{i}}{\partial x_{k}}+\frac{\partial u_{k}}{\partial x_{i}}\right)$. The governing equations are discretized, using second-order accuracy, on grids such as those discussed below, according to the cell-centered finite-volume method. For periodic flows, such as those studied in this paper, the flow solver performs sufficient number of real-time steps in order to reach periodic results. A parallel colored Gauss-Siedel method is used for solving the discretized equations in pseudo-time in each real time step.

In the cut-cell method this paper is dealing with, 3D grid generation is a challenging task. The use of an octree data structure makes it fast, with low memory requirements. Starting from a uniform Cartesian grid covering the entire flow domain, each cell intersected by the boundaries is recursively subdivided into eight sub-cells until the minimum allowed size is reached. By requiring that, each cell has at most four neighboring cells per face, the formation of big cells next to much smaller ones is avoided. Next step is to compute the exact intersection of cut-cells and the geometry surface [7]. Parts of cells inside the solid body are discarded yielding polyhedral cut-cells (Fig. 1).

The flow simulation might present convergence and/or accuracy issues if very small cut-cells appear next to much bigger cells. These should merge to form hyper finite volumes. At the end of this process, geometrical quantities required by the flow solver, such as the cell volume or barycentric coordinates, are computed. Cut- (generally, polyhedra) and uncut-cells
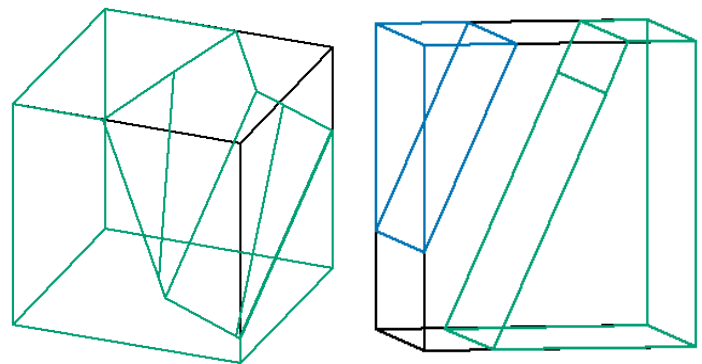

Fig. 1 Left: a cube (black lines) is intersected by the immersed body surface, creating the polyhedral volume (green lines); right: another cube (black lines) is separated into two different polyhedra (blue and green lines) by the two sides of an immersed body. 
(hexahedra) which entirely lie within the flow domain are treated differently. To compute the required geometrical quantities of the cut-cells, these are first subdivided into pyramids and their volumes and barycenters are analytically computed. For cells which are not cut by the boundaries, geometrical quantities are computed using a vector of three integers defining their position in the octree data structure [3]. This allows the direct computation of all geometrical quantities and facilitates access to neighboring cells. Moreover, the storage of all data as integers noticeably reduces memory usage.

The diaphragm motion is taken into account in the discretization of the flow equations, by applying the Reynolds theorem to compute the "physical" time derivative. Moreover, the Cartesian grid adaptation follows the geometry motion at each time-instant by coarsening or refining in areas far from and close to the boundaries respectively (Fig. 2). At each time-instant, flow quantities are transferred to the new grid, according to a conservative scheme. Changes in the shape of cut-cells and/or changes in the status of cells (from fluid to solid and vice-versa) are considered.
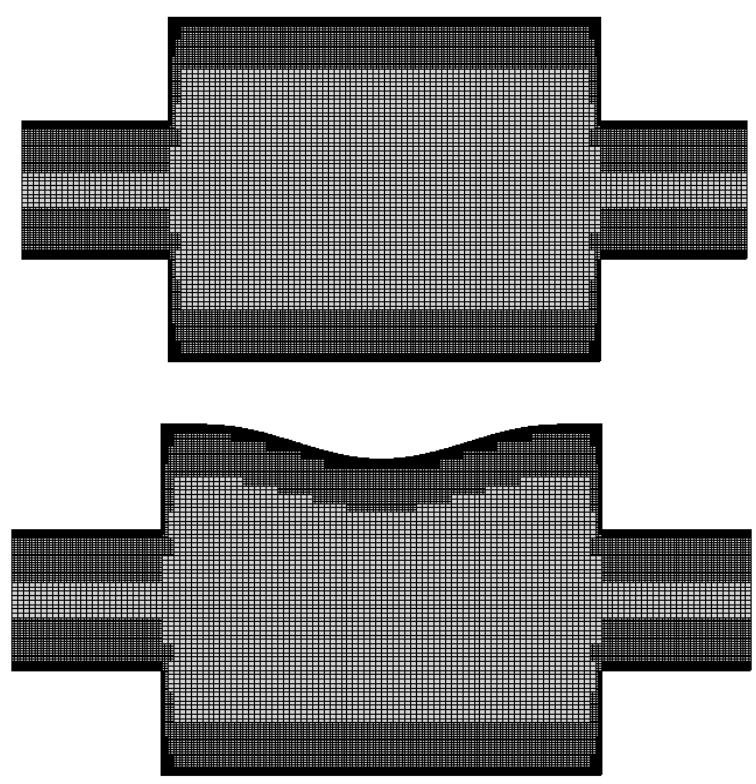

Fig. 2 Instantaneously adapted Cartesian grid over the symmetry plane of the diaphragm micropump. Cut and uncut cells within the fluid domain are only shown.

\section{The Hybrid Optimization Method}

The two-objective optimization carried out in this paper aims at minimizing the backflow at the pumps outlet diffuser while maximizing the net flowrate. The used optimization tool is hybrid, in the sense that it combines a stochastic population-based method (Metamodel-Assisted EA or MAEA) and the GB improvement of some optimal solutions [4]. An $(\mu, \lambda)$ MAEA, with $\mu$ parents and $\lambda$ offspring in each generation, is the background stochastic optimization tool. Metamodels (Radial Basis Function-RBF networks) assist the EA, by replacing as often as possible the use of the costly CFD tool. RBF networks are trained on individuals evaluated on the CFD tool during the evolution (on-line training) and predict the objective function values of new individuals at negligible cost. During the first generation, metamodels are inactive, waiting for enough training patterns to be collected. After that, each population member is pre-evaluated on its own locally trained metamodels and only the few most promising of them are re-evaluated on the CFD tool. Based on our experience, an MAEA is much faster, even by an order of magnitude or so, than a plain EA. Moreover, the kernel principal component analysis (PCA) method contributes to an additional cost reduction, as explained in details in Ref. [4]. The PCA of the current offspring population is performed anew in each generation and helps transform individuals from the design to the feature space in which the evolution operators perform better. Additionally, the PCA selects the most significant variables which the metamodels are trained on to reduce the training cost and increase their prediction ability.

On the other hand, a GB optimization method computes the gradient of a scalar function $(\widehat{F})$, defined as the weighted sum of the two aforementioned objectives, with respect to (w.r.t.) the design variable $b_{i}$. This is used to update the values of the latter by marching in the steepest descent direction, as follows (assuming a minimization problem): 


$$
b_{i}{ }^{\text {new }}=b_{i}{ }^{\text {old }}-\left.\eta \frac{\delta \hat{F}}{\delta b_{i}}\right|^{\text {old }}
$$

where $\eta$ is a user-defined step. To compute the gradient of $\hat{F}$ at a cost that is independent of the number of design variables, the continuous adjoint method [5] is used. In the adjoint method, the whole gradient is computed at about the cost of solving the flow equations once more. The objective function $\hat{F}$ (to be minimized) is augmented by the space/time integral of the product of the residuals of the flow equations and the adjoint variable fields (Lagrange multipliers, $\left.\Psi_{n}\right)$, namely,

$$
\hat{F}^{\text {aug }}=\hat{F}+\int_{0}^{T} \int_{\Omega} \Psi_{n} R_{n} d \Omega d t
$$

where $\Omega$ is the computational domain and $T$ is the (fixed) period. $\hat{F}^{a u g}$ is differentiated w.r.t. $b_{i}$ and the multipliers of the derivatives of the flow variables w.r.t. $b_{i}$ are set to zero, leading to an adjoint problem to be solved; the development of the adjoint equations and their boundary conditions can be found in Ref. [5]. The expression computing the gradient of $\hat{F}$ is formed by the remaining terms. The adjoint equations read,

$$
\begin{aligned}
R_{m}^{a}=\Gamma_{n m}^{-1} \frac{\partial \Psi_{n}}{\partial \tau} & -\frac{\partial \bar{\Psi}_{m}}{\partial t}-A_{n m k} \frac{\partial \Psi_{n}}{\partial x_{k}}-\frac{\partial \tau_{k m}{ }^{a}}{\partial x_{k}} \\
& =0
\end{aligned}
$$

where $\vec{\Psi}=\left[0 \Psi_{2} \Psi_{3} \Psi_{4}\right]^{T}, A_{n m k}$ stands for the Jacobian of the inviscid fluxes w.r.t. $\vec{V}$ and $\tau_{k m}^{A}$ are the adjoint stresses. The adjoint equations is discretized similarly to the flow equations and integrated backwards in time.

Upon the numerical solution of the adjoint equations, the gradient of $\hat{F}$ can be computed as

$$
\frac{\delta \hat{F}}{\delta b_{i}}=\int_{T} \int_{S_{d}}\left(\Psi_{1} n_{k}-\tau_{k m}^{a} n_{m}\right) \frac{\delta u_{k}^{d}}{\delta b_{i}} d S d t
$$

where $S_{d}$ is the diaphragm's surface, $\vec{n}$ is the normal vector to it and $u_{k}^{d}$ is its velocity. In a recent paper [4] by the same group, a hybrid optimization method that combines the advantages of MAEAs and GB methods has been presented. This method is employed in this paper as well. It combines the ability of EAs to compute Pareto fronts in problems with more than one objective with that of GB methods to improve candidate solutions in a straightforward manner provided that gradients have been computed. Their hybridization increases further the MAEA's efficiency, which is already much faster than that of a standard EA. The MAEA explores the design space, while the GB method regularly improves/refines a small user-defined number of the most promising individuals, which, in multi-objective optimization, belong to the current front of non-dominated solutions. In Ref. [4], a technique to compute "appropriate" descent directions for each individual to be refined by the GB method has been proposed. This results from the concatenation of the gradients of both objective functions into a single direction, referred to as the Pareto advancement direction (PAD). The PAD always points towards the simultaneous improvement of all objective functions. To compute it, the PCA of the objective function values of the current Pareto members in each generation is performed; this computes the so-called principal components of the objective space. The principal component with the smallest variance is considered to be "perpendicular" to the processed front and, thus, becomes the PAD; by moving in the PAD direction, all objective functions are improved. In each generation, after updating the PAD, a few individuals on the current front undergo GB improvement and the resulting solutions are inserted into the new offspring population. Fig. 3 depicts how the GB method updates, between two (arbitrarily selected) successive generations, the front of non-dominated solutions.

\section{Parameterization-Optimization}

The valveless micropump optimized/re-designed in this paper is based on an existing device ("reference" micropump), equipped with identical inlet and outlet diffusers (Fig.4). The chamber volume is $\sim 40 \mathrm{~mm}^{3}$ with $0.5 \mathrm{~mm}$ height and $\sim 8.9 \mathrm{~mm}$ length (excl. 


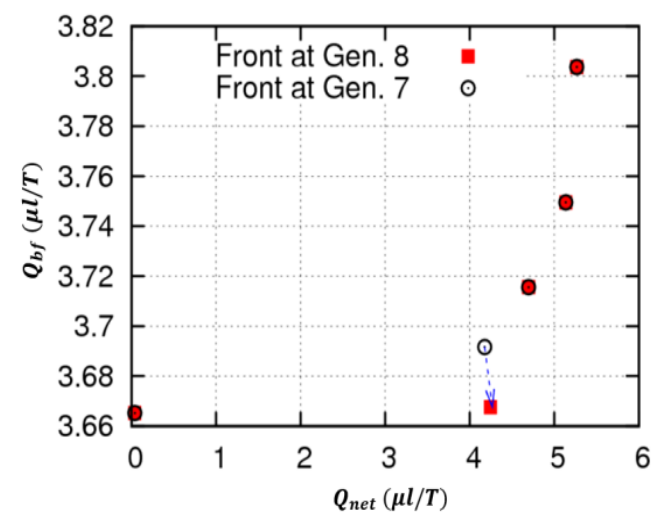

Fig. 3 PAD (blue arrow) upgrading the front from generation 7 to 8. Only part of the front is shown. Both axes correspond to volume flowrate per period.

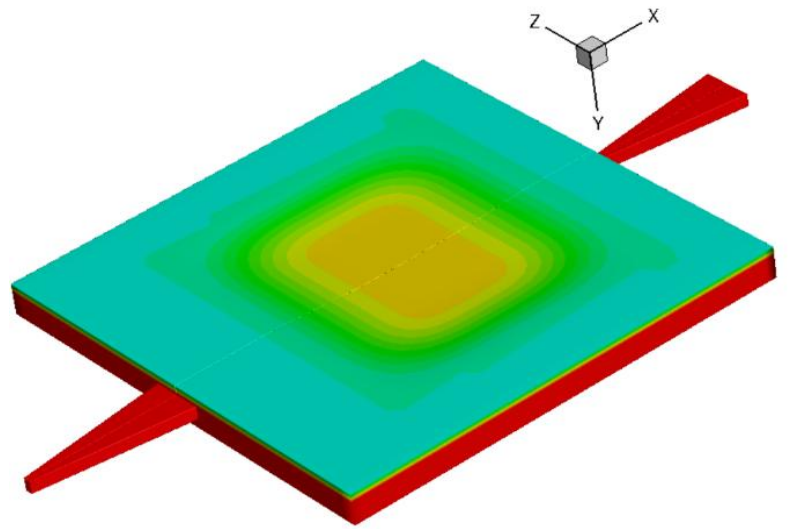

Fig.4 Perspective view of the valveless diaphragm micropump. Fluid flows from left to right.

diffusers), the inlet rectangular cross-sectional area is $\sim 0.03 \mathrm{~mm}^{2}$ and the outlet $\sim 0.2 \mathrm{~mm}^{2}$. The diaphragm (and therefore the fluid) is pushed from the inlet to the outlet with a predetermined frequency $(50 \mathrm{~Hz})$.

The diaphragm's motion parameterization defines 8 design variables. The area of the diaphragm is defined by two of them, namely $\left(b_{1}, b_{2}\right),\left(b_{3}, b_{4}\right)$ control the maximum displacement over all time instants through equation:

$$
y_{\max }(t)=b_{3} \exp \left(-b_{4}(t-T)^{2}\right)\left(1-\left|\frac{2 t}{T}\right|\right)
$$

where $T=0.02 \mathrm{sec}$ is the fixed period. The longitudinal (along $\mathrm{x}$-axis) deformation over time is controlled by $\left(b_{5}, b_{7}\right)$ and the span-wise (along the z-axis) deformation by $\left(b_{6}, b_{8}\right)$. Different time instants of the diaphragm deformation for the reference micropump can be seen in Fig. 5.

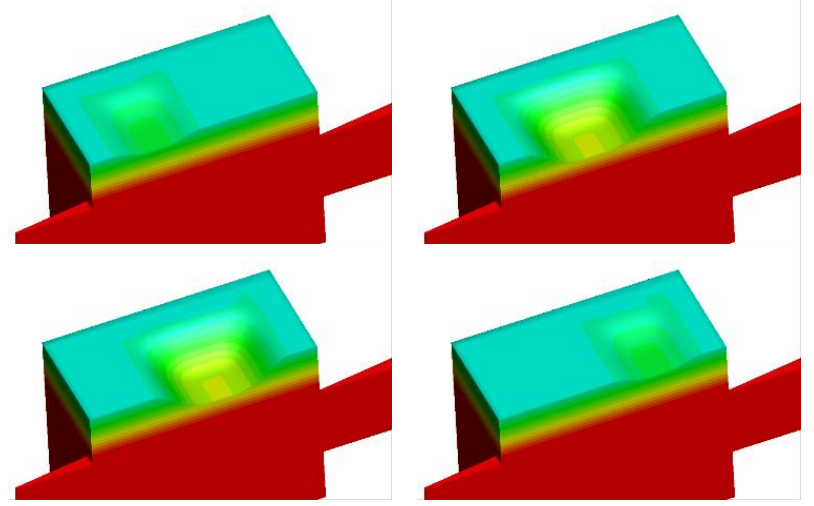

Fig. 5 Reference diaphragm motion. Diaphragm shapes/strokes at $t=0.25 \mathrm{~T}, 0.4 \mathrm{~T}, 0.6 \mathrm{~T}$, and $0.75 \mathrm{~T}$, from top-left to bottom-right, respectively. Axes not in scale.

The reference micropump delivers $Q_{n e t}=3 \mu \mathrm{L} / \mathrm{T}$ of fluid with a non-negligible backflow rate $\left(Q_{b f}=24 \mu \mathrm{L} / \mathrm{T}\right)$ though. Therefore, it was decided to run a two-objective optimization aiming at maximum $Q_{n e t}$ and minimum $Q_{b f}$. Aiming at minimum $\left|Q_{\text {net }}-Q_{\text {net,ref }}\right|$ could be an alternative objective. For the latter, the Pareto front could be derived by just post-processing the front of optimal solutions computed with the objective functions this paper is dealing with. Both $Q_{n e t}$ and $Q_{b f}$ are measured in $\mu \mathrm{L}$ per period $(\mu \mathrm{L} / \mathrm{T})$. A $(6,12)$ MAEA is the basic search method, with metamodels and the PCA technique activated just after the first generation. The GB method updates only two individuals per generation, by performing a single descent step for each of them; the required gradients are computed by the adjoint method. The computational budget is limited to 150 CFD runs with 44 of them spent for the solution of adjoint problems. Fig. 8 shows the computed Pareto front of non-dominated solutions.

Fig. 7 shows how the backflow and net volume flux evolve over time for the two edges of the Pareto front and the reference micropump. Great differences between solutions A and B can be seen. In solution $\mathrm{A}, Q_{b f}$ has been reduced a lot and takes place only during a very small percentage of the period.

Even if the manufacturability of the diaphragm and the mechanism controlling its motion are beyond the 

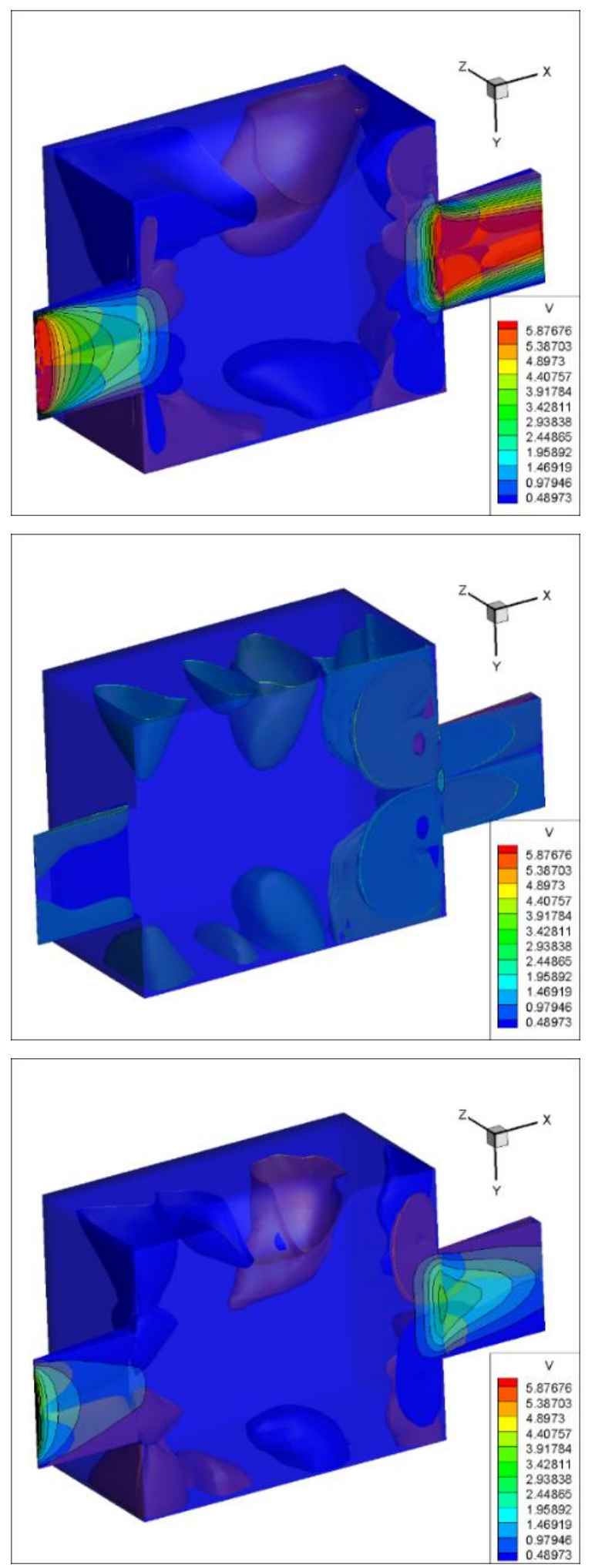

Fig. 6 Velocity magnitude iso-areas on the symmetry plane at $t=0.5 \mathrm{~T}$. Vorticity iso-surfaces within the 3D volume are also shown. Reference (top), min. $Q_{b f}$ (middle) and max. $Q_{\text {net }}$ solutions (bottom). Axes not in scale, y-axis is 14 times greater.
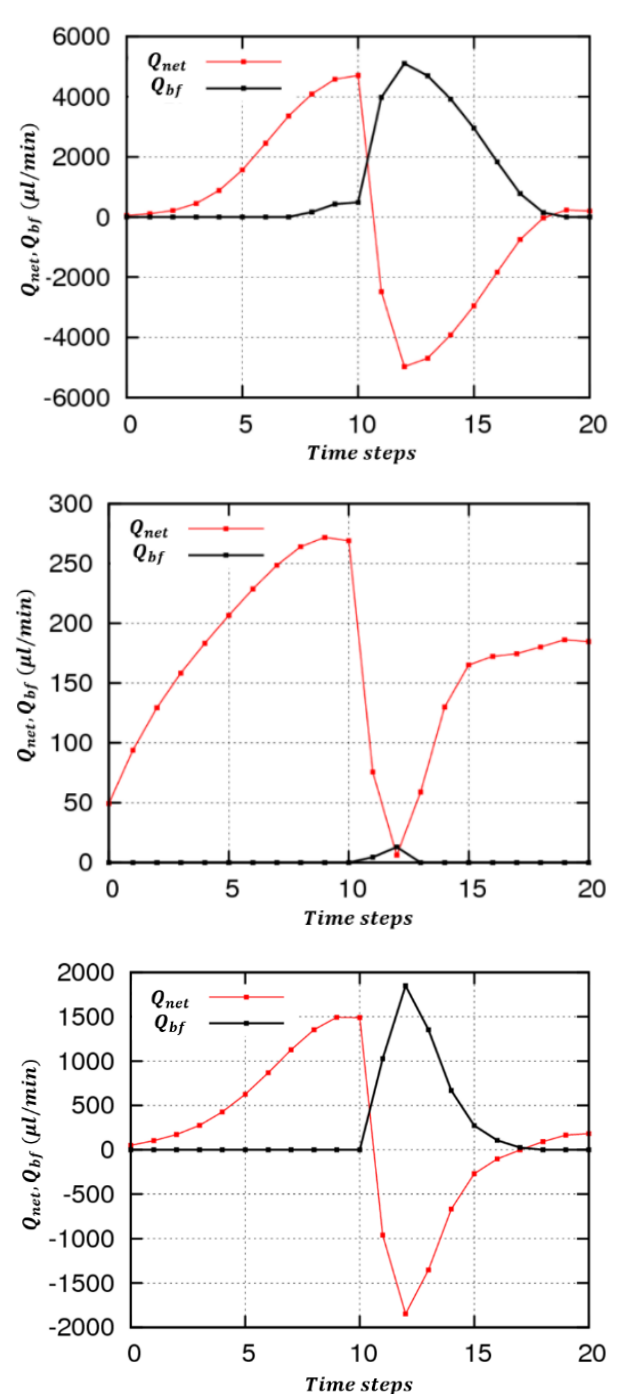

Fig. $7 Q_{b f}$ and $Q_{n e t}$ time-series for the reference (top), min. $Q_{b f}$ (middle) and max. $Q_{n e t}$ motion (bottom). The length of the horizontal axis corresponds to a period $T=$ 0.02 sec, discretized with 20 time steps.

scope of this paper, it was deemed necessary to include also a study on the effect uncertainties may have on the performance of micropumps with optimal diaphragm motion; the examined cases are the two edges of the Pareto front. Uncertainties are associated with all design variables, by assuming mean values $(\mu)$ and standard deviations $(\sigma)$ for each of them: $\mu$ is set equal to the computed values of the design variables for this optimal solution and $\sigma$ to the $1 \%$ of this value.

The non-intrusive PCE method [11] is used to propagate these uncertainties to the two objective 
functions, $Q_{n e t}$ and $Q_{b f}$ (uncertainty quantification, UQ). In the PCE method, all uncertain variables are expanded in suitable series and probability density functions and orthogonal polynomials are associated with them. The first two statistical moments are expressed in terms of the coefficients of this expansion. Based on the orthogonality properties of the above polynomials, the unknown expansion coefficients (and, thus, the sought statistical moments) are computed by Galerkin projections. This involves integral expressions which are computed using the Gaussian integration formulas, by defining Gaussian nodes in the uncertain space and performing CFD evaluations for them. However, the non-intrusive variant of the PCE method suffers from the curse of dimensionality. For instance, here, with 8 uncertain variables, the cost of a single UQ becomes quite expensive, for a second-order chaos expansion. To avoid this very costly sampling, Smolyak sparse grids [8] have been used; in practice, in our problem, the UQ of a single micropump costs as many as $17 \mathrm{CFD}$ evaluations on the cut-cell method, instead of 256 required by the Gaussian integration method. The outcome of the UQ study is also shown in Fig. 8, where the mean values of the two objective functions

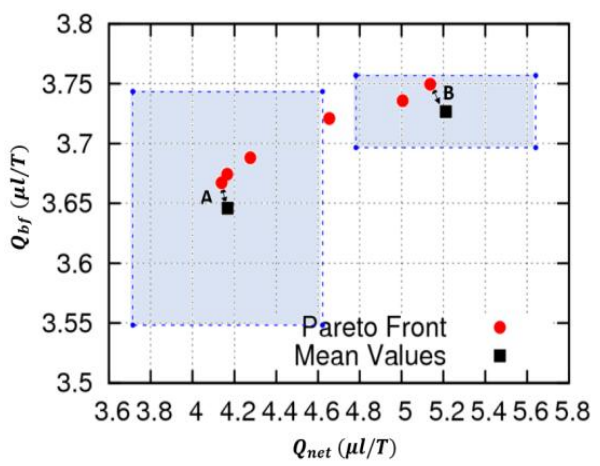

Fig. 8 Computed front of non-dominated solutions. Mean value and standard deviations of the min. $Q_{b f}$ (point A) and max. $Q_{\text {net }}$ (point B) motion, plotted in the objective space with black filled squares and blue dotted boxes. The point corresponding to the objective function values ( $Q_{b f}=24 \mu l / T \& Q_{n e t}=3 \mu l / T$ ) for the reference diaphragm motion can not be represented within the selected graph limits. Regarding the UQ results, comments can be found in the results section. (black squares) and the $\pm 3 \sigma$ (here, $\sigma$ is the standard deviation of the objective function) area (dotted quadrilaterals) around them are plotted. The solution with the lowest backflow seems to be exposed to a higher uncertainty regarding the backflow itself.

\section{Conclusions}

The purpose of this paper is to redesign/optimize a valveless diaphragm micropump for medical applications, using a hybrid optimization method. This includes a gradient-free population-based search that acts as the background search tool with the selective implementation of gradient-based refinement of some of the optimal solutions. Having the extension of this work to valved diaphragm pumps with rotating valves in mind (on-going research), it was decided to use an in-house 3D cut-cell CFD method as the evaluation tool. To compute the gradient of the objective function(s), the continuous adjoint method was mathematically formulated, programmed and used. By selecting the net flowrate and the amount of backflow per period as the two objective functions, the Pareto front of non-dominated solutions has been found. It is important to mention that all front members clearly dominate the existing micropump. To reduce the backflow, being quite critical in most medical applications, light strokes leading to minimal diaphragm motion are necessary; in contrast, to increase the net flowrate per period, more intensive strokes on a smaller part of the diaphragm are necessary. To account for manufacturing or operating uncertainties, the uncertainty quantification of the performance of some optimal solutions was finally deemed necessary. It was found that uncertainties in the operating conditions seriously affect backflow.

\section{Acknowledgment}

We acknowledge support of this work by the project "Design-Optimization of Diaphragm Pumps under Operational/Manufacturing Uncertainties Using the Cut-Cell Method and Polynomial Chaos 
Expansion" (MIS 5004541) which is implemented under the action "Supporting Researchers with an Emphasis on Young Researchers", in the context of the call EDBM34, funded by the Operational Programme "Human Resource Development, Education and Lifelong Learning" (NSRF 2014-2020) and co-financed by Greece and the European Union (European Regional Development Fund).

\section{References}

[1] Bar-Cohen, Y., and Chang, Z. 2000. "Piezoelectrically Actuated Miniature Peristaltic Pump." In Proceedings of SPIE's 7th Annual International Symposium on Smart Structures and Materials, 92-103.

[2] Chun-Wei, H., Song-Bin, H., and Gwo-Bin, L. 2008. "A Microfluidic Device for Precise Pipetting." Journal of Micromechanics and Microengineering 18: 35-9.

[3] Ji, H., Lien, F., and Yee, E. 2010. "A New Adaptive Mesh Refinement Data Structure with an Application to Detonation." Journal of Computational Physics 229: 8981-93.

[4] Kapsoulis, D., Tsiakas, K., Trompoukis, X., Asouti, V., and Giannakoglou, K. 2018. "A PCA-Assisted Hybrid Algorithm Combining EAs and Adjoint Methods for CFD-Based Optimization." Applied Soft Computing 73:
520-9.

[5] Papoutsis-Kiachagias, E., and Giannakoglou, K. 2016. "Continuous Adjoint Methods for Turbulent Flows, Applied to Shape and Topology Optimization: Industrial Applications." Archives of Computational Method in Engineering 23: 255-99.

[6] Samouchos, K., Kapsoulis, D., Trompoukis, X., and Giannakoglou, K. 2019. "Shape Optimization of 3D Diaphragm Pumps Using the Continuous Adjoint Approach to the Cut-Cell Method." Presented at the ICCM Congress, Singapore.

[7] Samouchos, K., Katsanoulis, S., and Giannakoglou, K. 2016. "Unsteady Adjoint to the Cut-Cell Method Using Mesh Adaptation on GPUs." Presented at the ECCOMAS Congress, Crete Island, Greece.

[8] Smolyak, S. 1963. "Quadrature and Interpolation Formulas for Tensor Products of Certain Classes of Functions." Dokl. Akad. Nauk SSSR 4: 240-3.

[9] Stremme, E., and Stremme, G. 1993. "A Valveless Diffuser/Nozzle-Based Fluid Pump." Sensors and Actuators A: Physical 39: 159-67.

[10] Wackerle, M., Bigus, H., and Blumenthal, T. 2006. Micro Pumps for Lab Technology and Medicine. Munich: Fraunhofer IZM.

[11] Xiu, D., and Karniadakis, G. 2003. "Modeling Uncertainty in Flow Simulations via Generalized Polynomial Chaos.” Journal of Computational Physics 187: 37-67. 